\title{
Leptonic decays of the tau lepton
}

\author{
Matteo Fael ${ }^{1, *}$ \\ ${ }^{1}$ Theoretische Physik I, Universität Siegen, Walter-Flex-Strasse 3, 57068 Siegen, Germany
}

Abstract. In these proceedings we review the SM prediction for the tau leptonic decays, including the radiative $(\tau \rightarrow \ell \gamma v \bar{v})$ and the five-body $\left(\tau \rightarrow \ell \ell^{\prime} \ell^{\prime} v \bar{v}\right)$ decay modes, which are among the most powerful tools to study precisely the structure of the weak interaction and to constrain possible contributions beyond the V-A coupling of the Standard Model.

\section{Introduction}

The leptonic decays of the tau are among the most effective tools to study the structure of the weak interaction the Standard Model (SM) and possible hints of new physics via the Michel parameters [1-4]. Michel parameters can be measured not only in three-body decays $(\tau \rightarrow \ell \nu \bar{v})$ but also in muon and tau radiative modes [5-8],

$$
\begin{aligned}
\mu & \rightarrow e v \bar{v} \gamma, \\
\tau & \rightarrow \ell v \bar{v} \gamma, \text { with } \ell=\mu, e,
\end{aligned}
$$

and in the rare five-body decays [9]

$$
\begin{aligned}
\mu & \rightarrow e e e v \bar{v}, \\
\tau & \rightarrow \ell \ell^{\prime} \ell^{\prime} v \bar{v}, \text { with } \ell, \ell^{\prime}=\mu, e .
\end{aligned}
$$

The BABAR and BELLE collaborations measured the $\tau \rightarrow \ell \gamma \nu \bar{v}$ branching ratios for a minimum photon energy of $\omega_{0}=10 \mathrm{MeV}$ in the tau rest frame $[8,10]$. These measurements, with a relative error of about 3\% (BABAR) and 6\% (BELLE), must be compared with the SM predictions at next-to-leading order (NLO). Indeed these radiative corrections are not protected from mass singularities by Kinoshita-Lee-Nauenberg (KLN) theorem [11, 12] and are of relative order $(\alpha / \pi) \ln \left(m_{\ell} / m_{\tau}\right) \ln \left(\omega_{0} / m_{\tau}\right)$, corresponding to a $10 \%$ correction for $\ell=e$, and $3 \%$ for $\ell=\mu$. A study of five-body leptonic decays at BELLE is ongoing with a data sample of about $0.91 \times 10^{9} \tau^{+} \tau^{-}$pairs [13-15].

These leptonic modes are also an important source of background to Charge Lepton Flavour Violation (CLFV) searches. The radiative decays $(1,2)$ can mimic the SM forbidden processes $\mu \rightarrow e \gamma$ and $\tau \rightarrow \ell \gamma$, while the five-body decays $(3,4)$ are among the background of $\mu \rightarrow$ eee conversion at the Mu3E experiment [16], and $\tau \rightarrow \ell \ell^{\prime} \ell^{\prime}$ conversion at $e^{+} e^{-}$colliders. Indeed they are all indistinguishable from the CLFV signal except for the energy carried out by neutrinos.

These proceedings review the NLO predictions for the tau and muon radiative decays [17, $18]$ and five-body decays $[19,20]$. We also report the NLO corrections to the branching ratios for the tau decays (4), omitted in Refs. [19, 20].

\footnotetext{
*e-mail: fael@physik.uni-siegen.de
} 


\section{Details of the calculation}

We adopted the Fermi $V-A$ effective theory of weak interactions:

$$
\mathcal{L}=\mathcal{L}_{\mathrm{QED}}+\mathcal{L}_{\mathrm{QCD}}-\frac{4 G_{F}}{\sqrt{2}}\left(\bar{\psi}_{v_{\tau}} \gamma^{\alpha} P_{L} \psi_{\tau}\right) \cdot\left(\bar{\psi}_{\ell} \gamma_{\alpha} P_{L} \psi_{v_{\ell}}\right)+\text { h.c. },
$$

with $\ell=\mu, e$ and where $\psi_{\tau}, \psi_{\mu}, \psi_{e}, \psi_{v_{\tau}}, \psi_{v_{\mu}}, \psi_{v_{e}}$ are the fields of tau, muon, electron and their associated neutrinos, respectively; $P_{L}=\left(1-\gamma_{5}\right) / 2$ is the left-hand projector. A Fierz rearrangement of the four-fermion interaction (5) allows to factorize the amplitudes into the product of spinor chains depending either on the neutrino momenta or on the muon and electron ones (see Appendix A.3 in [19]), so that the neutrino's phase space integration can be done analytically.

The one-loop amplitudes are reduced to tensor integrals with Form [21] and the Mathematica package FeynCALC [22, 23], then exported to Fortran for their numerical integration. Our code uses LoopTools [24, 25] as well as Collier [26] for the computation of the one-loop tensor coefficients, which can be both employed and compared. The numerical integrations are performed with a standard Monte Carlo via the Vegas [27] algorithm in CuBa [28].

Ultraviolet divergences are regularized via dimensional regularization and renormalized in the on-shell scheme. A small photon mass is introduced to regularize the infrared (IR) divergences, while the finite electron and muon masses regularize the collinear ones. In order to handle the IR divergences, we adopted a phase-space slicing method and as well as the QED dipole subtraction [29] to improve numerical stability of our code when dealing with the tau five-body decays.

\section{The radiative decays}

\begin{tabular}{lrrrr}
\hline & $\tau \rightarrow e \bar{v} \gamma \gamma$ & $\tau \rightarrow \mu \bar{\nu} \gamma \gamma$ & $\mu \rightarrow e v \bar{v} \gamma$ & $\mu \rightarrow e v \bar{\gamma} \gamma$ \\
\hline $\mathcal{B}_{\mathrm{LO}}$ & $1.834 \cdot 10^{-2}$ & $3.663 \cdot 10^{-3}$ & $1.308 \cdot 10^{-2}$ & $6.204 \cdot 10^{-8}$ \\
$\mathcal{B}_{\mathrm{NLO}}^{\text {Inc }}$ & $-1.06(1)_{n}(10)_{N} \cdot 10^{-3}$ & $-5.8(1)_{n}(2)_{N} \cdot 10^{-5}$ & $-1.91(5)_{n}(6)_{N} \cdot 10^{-4}$ & $-3.61(8)_{n}(21)_{N} \cdot 10^{-9}$ \\
$\mathcal{B}_{\mathrm{NLO}}^{\mathrm{Exc}}$ & $-1.89(1)_{n}(19)_{N} \cdot 10^{-3}$ & $-9.1(1)_{n}(3)_{N} \cdot 10^{-5}$ & $-2.25(5)_{n}(7)_{N} \cdot 10^{-4}$ & $-3.61(8)_{n}(21)_{N} \cdot 10^{-9}$ \\
$\mathcal{B}^{\text {Inc }}$ & $1.728(10)_{\mathrm{th}}(3)_{\tau} \cdot 10^{-2}$ & $3.605(2)_{\mathrm{th}}(6)_{\tau} \cdot 10^{-3}$ & $1.289(1)_{\mathrm{th}} \cdot 10^{-2}$ & $5.84(2)_{\mathrm{th}} \cdot 10^{-8}$ \\
$\mathcal{B}^{\mathrm{Exc}}$ & $1.645(19)_{\mathrm{th}}(3)_{\tau} \cdot 10^{-2}$ & $3.572(3)_{\mathrm{th}}(6)_{\tau} \cdot 10^{-3}$ & $1.286(1)_{\mathrm{th}} \cdot 10^{-2}$ & $5.84(2)_{\mathrm{th}} \cdot 10^{-8}$ \\
\hline
\end{tabular}

Table 1. Branching ratios of radiative $\mu$ and $\tau$ leptonic decays. The minimum photon energy $\omega_{0}$ is $10 \mathrm{MeV}$, except for the last column, where $\omega_{0}=40 \mathrm{MeV}$ and $E_{e}^{\mathrm{min}}=45 \mathrm{MeV}$. Inclusive and exclusive $\left(\mathcal{B}^{\text {Inc/Exc }}\right)$ predictions are separated into LO contributions $\left(\mathcal{B}_{\mathrm{LO}}\right)$ and NLO corrections $\left(\mathcal{B}_{\mathrm{NLO}}^{\mathrm{Inc} / \mathrm{Exc}}\right)$. Uncertainties were estimated for uncomputed NNLO corrections $(N)$, numerical $(n)$ and the experimental errors of the lifetimes $(\tau)$. The first two were combined into a total uncertainty (th).

At NLO, which allows for double photon emission, the branching ratios of the radiative decays (1) and (2) can be distinguished in two types:

Inclusive: measurement of the branching ratios, $\mathcal{B}_{\text {Inc }}\left(\omega_{0}\right)$, where at least one photon in the final state has an energy grater than $\omega_{0}$.

Exclusive: measurement of the branching ratios, $\mathcal{B}_{\mathrm{Exc}}\left(\omega_{0}\right)$, where one, and only one photon in the final state has an energy grater than $\omega_{0}$. 
Exclusive and inclusive branching ratios for the radiative decays were computed in [17] and [18] for a threshold $\omega_{0}=10 \mathrm{MeV}$, and are reported in Tab. 1. Uncertainties were estimated for unknown NNLO corrections, numerical errors, and the experimental errors of the lifetimes. For $\omega_{0}=10 \mathrm{MeV}$, the former were estimated to be $\delta \mathcal{B}_{\mathrm{NLO}}^{\mathrm{Exc} / \mathrm{Inc}} \sim$ $(\alpha / \pi) \ln \left(m_{\ell} / m_{\tau}\right) \ln \left(\omega_{0} / m_{\tau}\right) \mathcal{B}_{\mathrm{NLO}}^{\mathrm{Exc} / \mathrm{Inc}}:$ about $10 \%, 3 \%$ and $3 \%$ for $\tau \rightarrow e \bar{v} v \gamma, \tau \rightarrow \mu \bar{\nu} v \gamma$ and $\mu \rightarrow e \bar{\nu} v \gamma$, respectively (they appear with the subscript " $N$ " in Tab. 1). Numerical errors $(n)$ are smaller than those induced by missing radiative corrections. These two kinds of uncertainties were combined to provide the total theoretical error of $\mathcal{B}^{\mathrm{Exc} / \mathrm{Inc}}$ (th). The uncertainty due to the experimental error of the lifetimes $(\tau)$ is also reported.

BABAR and BELLE measurements of the branching ratios of (2), for a minimum photon energy $\omega_{0}=10 \mathrm{MeV}$ in the $\tau$ rest frame, are $[8,10]$ :

$$
\begin{aligned}
\mathcal{B}_{\text {BABAR }}(\tau \rightarrow e \bar{v} v \gamma) & =1.847(15)_{\mathrm{st}}(52)_{\mathrm{sy}} \times 10^{-2}, \\
\mathcal{B}_{\text {BABAR }}(\tau \rightarrow \mu \bar{v} v \gamma) & =3.69(3)_{\mathrm{st}}(10)_{\mathrm{sy}} \times 10^{-3} \\
\mathcal{B}_{\text {Belle }}(\tau \rightarrow e \bar{v} v \gamma) & =1.79(2)_{\mathrm{st}}(10)_{\mathrm{sy}} \times 10^{-2} \\
\mathcal{B}_{\text {Belle }}(\tau \rightarrow \mu \bar{v} v \gamma) & =3.63(2)_{\mathrm{st}}(15)_{\mathrm{sy}} \times 10^{-3}
\end{aligned}
$$

They are substantially more precise than the previous measurements by CLEo [30]. The experimental values in Eqs. (6-9) were obtained requiring a signal with either a muon or an electron, plus a single photon; they must therefore be compared with the exclusive branching ratios in Tab. 1. For $\tau \rightarrow \mu \bar{v} v \gamma$ decays, the BABAR and Belle measurements and prediction agree within $1.1 \sigma$ and $0.4 \sigma$, respectively. On the contrary, the BABAR's values for $\tau \rightarrow e \bar{v} v \gamma$ differs by $2.02(57) \times 10^{-3}$, i.e. by $3.5 \sigma$. In [18] it was showed showed that very plausibly the $3.5 \sigma$ discrepancy between the BABAR measurement of $\mathcal{B}(\tau \rightarrow$ e $\gamma v \bar{v})$ and the NLO result is related to not using a full NLO calculation when estimating the efficiencies.

The branching ratio of radiative muon decays was measured long ago for a minimum photon energy $\omega_{0}=10 \mathrm{MeV}$ [31], and more recently by the MEG collaboration for $\omega_{0}=$ $40 \mathrm{MeV}$ and minimum electron energy $E_{e}^{\mathrm{min}}=45 \mathrm{MeV}$ (in this case, $\mathcal{B}^{\text {Inc }}$ and $\mathcal{B}^{\text {Exc }}$ coincide):

$$
\begin{aligned}
& \mathcal{B}_{\mathrm{EXP}}\left(\mu \rightarrow e \bar{v} v \gamma, \omega_{0}=10 \mathrm{MeV}\right)=1.4(4) \times 10^{-2} \quad[31] \\
& \mathcal{B}_{\mathrm{EXP}}\left(\mu \rightarrow e \bar{\nu} v \gamma, \omega_{0}=40 \mathrm{MeV}, \mathrm{E}_{\mathrm{e}}^{\min }=45 \mathrm{MeV}\right)=6.03(14)_{\mathrm{st}}(53)_{\mathrm{sy}} \times 10^{-8}
\end{aligned}
$$

Both measurements agree with our theoretical predictions (see Tab. 1). New precise results are expected from the Meg [32] and PiBETA [33] collaborations.

\section{The five-body decays}

The LO branching ratios and the NLO corrections for the five-body decays $(3,4)$ are presented in Tab. 2. The second column shows the branching fraction at LO, while the third and the fourth report separately the NLO contributions due to photons and leptons only (the dominant part) and the correction given by the hadronic vacuum polarization. The last column displays the shift of the LO branching ratio induced by radiative corrections. The uncertainties in Tab. 2 are the errors from numerical integration. On top of the quoted uncertainties, for the tau one must take into account also the error due to the tau lifetime; at present it corresponds to a fractional uncertainty $\delta \tau_{\tau} / \tau_{\tau}=1.7 \times 10^{-3}$ [34], which is of the same order of magnitude as the NLO corrections for the first two modes in Tab. 2. For the rare muon decay the error due to the lifetime is negligible.

The NLO corrections to the branching ratios are of order $0.1 \%$ for the tau decays involving at least two electrons (the first two modes in Tab. 2) and the five-body muon decay. 


\begin{tabular}{lrrrr}
\hline & \multicolumn{1}{c}{$\mathcal{B}_{\mathrm{LO}}$} & \multicolumn{1}{c}{$\delta \mathcal{B}_{\text {lep }}$} & \multicolumn{1}{c}{$\delta \mathcal{B}_{\text {had }}$} & $\delta \mathcal{B} / \mathcal{B}$ \\
\hline$\tau \rightarrow e e e v \bar{v}$ & $4.2488(4) \times 10^{-5}$ & $-4.2(1) \times 10^{-8}$ & $-1.0 \times 10^{-9}$ & $-0.1 \%$ \\
$\tau \rightarrow \mu e e v \bar{v}$ & $1.989(1) \times 10^{-5}$ & $4.4(1) \times 10^{-8}$ & $-6.6 \times 10^{-10}$ & $0.2 \%$ \\
$\tau \rightarrow e \mu \mu \nu \bar{v}$ & $1.2513(6) \times 10^{-7}$ & $2.70(1) \times 10^{-9}$ & $-3.6 \times 10^{-10}$ & $1.8 \%$ \\
$\tau \rightarrow \mu \mu \mu \nu \bar{v}$ & $1.1837(1) \times 10^{-7}$ & $2.276(2) \times 10^{-9}$ & $-3.5 \times 10^{-10}$ & $1.6 \%$ \\
\hline$\mu \rightarrow e e e v \bar{v}$ & $3.6054(1) \times 10^{-5}$ & $-6.69(5) \times 10^{-8}$ & $-1.8 \times 10^{-11}$ & $0.2 \%$ \\
\hline
\end{tabular}

Table 2. LO and NLO branching ratios of $\tau \rightarrow \ell \ell^{\prime} \ell^{\prime} v \bar{v}$ (with $\ell, \ell^{\prime}=e, \mu$ ) and $\mu \rightarrow e e e v \bar{v}$. The NLO correction due to photons and leptons only is denoted by $\delta \mathcal{B}_{\text {lep }}$, while the non-perturbative contribution given by the hadronic vacuum polarization is denoted by $\delta \mathcal{B}_{\text {had }}$. The last column report the ratio between the NLO correction and the LO branching ratio. The uncertainties are the error due to numerical integration.

They are one order of magnitude larger for the tau decays into at least two muons (the third and fourth modes in Tab. 2). Such difference is caused by the running of the fine structure constant $\alpha$. In the decays $\tau \rightarrow e \mu \mu \nu \bar{v}$ and $\tau \rightarrow \mu \mu \mu \nu \bar{v}$ the off-shell photon that converts into $\mu^{+} \mu^{-}$is forced to acquire an invariant mass of at least twice the muon mass and therefore the electron's contribution to the photon vacuum polarization generates a logarithmic enhancement $\frac{\alpha}{3 \pi} \log \left(4 m_{\mu}^{2} / m_{e}^{2}\right)$, which can be reabsorbed into the redefinition of $\alpha$ by substituting $\alpha \rightarrow \alpha\left(4 m_{\mu}^{2}\right)$. Note indeed that the shift induced by the running of $\alpha$ is $2 \times \Delta \alpha\left(4 m_{\mu}^{2}\right)=1.2 \%$, of the same order as the NLO corrections. This does not contradict the KLN theorem, which guarantees that radiative corrections are free from mass singularities except for those that can be reabsorbed into the running of coupling constant.

The branching ratio of (3) was measured long time ago by the Sindrum experiment [35],

$$
\mathcal{B}_{\exp }\left(\mu^{-} \rightarrow e^{+} e^{-} e^{-} v_{\mu} \bar{v}_{e}\right)=3.4(4) \times 10^{-5},
$$

while for the tau five-body decays, the CLEo experiment measured [36]

$$
\mathcal{B}_{\exp }\left(\tau \rightarrow e e^{-} e^{+} v \bar{v}\right)=2.8(1.5) \times 10^{-5},
$$

and established for $\tau \rightarrow \mu e e v \bar{v}$ the upper bound $\mathcal{B}_{\exp }\left(\tau \rightarrow \mu e^{-} e^{+} v \bar{v}\right)<3.2 \times 10^{-5}$ at $90 \%$ C.L. . All available experimental measurements are in good agreement with the results in Tab. 2. BELLE is expected to present soon new measurements of the branching fractions for $\tau \rightarrow$ eeev $\bar{v}$ and $\tau \rightarrow \mu e e v \bar{v}$, and to report upper bounds for the other two modes [13-15].

\section{Impact on CLFV Searches.}

Branching ratios of five-body decays are protected from large logarithmic corrections by the KLN theorem. However selection cuts on the final state can enhance the role of radiative corrections even up to $10 \%$. As an example, we discuss here the size of these corrections in the specific final-state configuration of (3) where the neutrino missing energy $(\mathbb{E})$ is very small and the visible energy $\left(E_{\mathrm{vis}}\right)$ is close to $m_{\mu}$. This region is particularly important for the Mu3E experiment. Indeed, in this phase-space point the muon decay (3) becomes a source of time- and space-correlated background for the CLFV three-body decay $\mu \rightarrow$ eee.

Fig. 1(left) displays the normalized NLO differential rate as function of the three-electron invariant mass $m_{123}$, close to the end point $m_{123}=m_{\mu}$. The local $K$-factor is shown in the lower part. The rate, evaluated at fixed value of $m_{123}$, is fully inclusive in the bremsstrahlung photon contribution. Fig. 1(right) shows the branching ratio $\mathcal{B}_{\mathrm{NLO}}\left(\mathbb{E}_{\max }\right)$ versus the cut on 

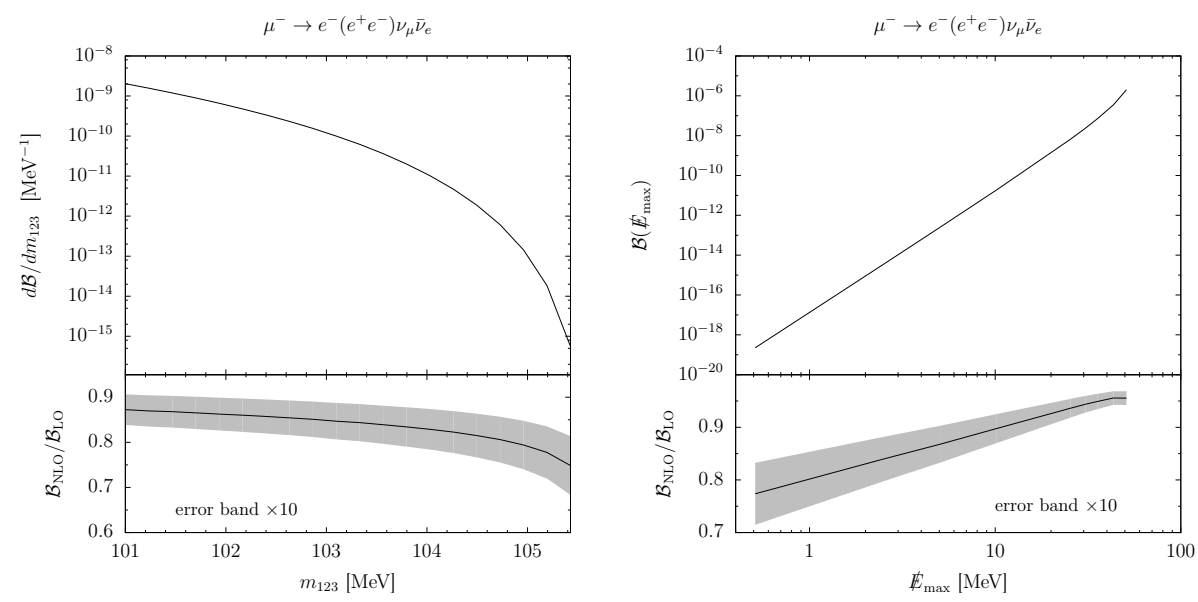

Figure 1. The branching ratio of $\mu \rightarrow e e e v \bar{v}$ at NLO as a function of the invariant mass of the three electrons $m_{123}$ (left) and the cut on the invisible energy $\mathbb{E}_{\max }$ (right). The ratio between the NLO and LO predictions is depicted in the lower part of each panel. The error band (magnified 10 times) represents the assigned theoretical error. Figures taken from [19].

the missing energy (upper panel) and its relative magnitude with respect to the LO prediction (lower panel). The branching ratio $\mathcal{B}\left(\mathbb{E}_{\max }\right)$ is calculated with a cut on the missing energy $\mathbb{E}=m_{\mu}-E_{\text {vis }} \leq \mathbb{E}_{\text {max }}$. Both distributions in Fig. 1 show that radiative corrections decrease the LO prediction by about $10-20 \%$, depending on the cut applied on the missing energy. Hence, the background events for $\mu \rightarrow$ eee due to the decay (3) are fewer than what is expected from a tree-level calculation.

\section{Conclusion}

We have reviewed the NLO predictions for the decay $\tau \rightarrow \ell \gamma v \bar{v}$ and $\tau \rightarrow \ell \ell^{\prime} \ell^{\prime} v \bar{v}$, with $\ell, \ell^{\prime}=e, \mu$ which were presented in [17-20]. The precise measurements by BABAR (BELLE) of the branching ratio $\mathcal{B}(\tau \rightarrow \mu \bar{\nu} v \gamma)$, for $\omega_{0}=10 \mathrm{MeV}$, agree with our prediction within $1.1 \sigma$ $(0.4 \sigma)$. On the contrary, BABAR's measurement of $\mathcal{B}(\tau \rightarrow e \bar{v} v \gamma)$, differs from our prediction by $3.5 \sigma$. This puzzling discrepancy deserves further researches.

Radiative corrections shift $\mathcal{B}\left(\tau \rightarrow \ell \ell^{\prime} \ell^{\prime} v \bar{v}\right)$ by about $0.1 \%$, for the modes with at least two electrons, and by $1 \%$ for the modes with at least two muons. These corrections are small because of cancellation of mass singularities in inclusive observables. The only logarithmic enhancement appearing in five-body decays is due too the running of the fine structure constant $\alpha$.

Detector acceptances and selection cuts can enlarge the magnitude of radiative corrections up to $10 \%$ level also for $\tau \rightarrow \ell \ell^{\prime} \ell^{\prime} v \bar{v}$. For instance the $\mu \rightarrow e e e v \bar{v}$ differential rate, when the total visible energy is close to the muon mass, is decreased by about $10-20 \%$. This corner of the phase space is of particular importance for the Mu3E experiment since the decay is a background process to the CLFV decay $\mu \rightarrow$ eee.

Acknowlefgements: A special thank to the organizers for the kind invitation and for the wonderful conference in Novosibirsk. This review is based on Refs. [17, 19], so I am indebted to my collaborators C. Greub and M. Passera. I also thank S. Eidelman, D. Epifanov, Y. Jin, G. M. Pruna, A. Signer, J. 
Sasaki and Y. Ulrich for very useful discussions and correspondence. This work was supported by Swiss National Science Foundation and by DFG through the Research Unit FOR 1873 "Quark Flavour Physics and Effective Field Theories".

\section{References}

[1] L. Michel, Proc. Phys. Soc. A63, 514 (1950), [,45(1949)]

[2] C. Bouchiat, L. Michel, Phys. Rev. 106, 170 (1957), [,89(1957)]

[3] T. Kinoshita, A. Sirlin, Phys. Rev. 107, 593 (1957)

[4] T. Kinoshita, A. Sirlin, Phys. Rev. 108, 844 (1957)

[5] W. Eichenberger, R. Engfer, A. Van Der Schaaf, Nucl. Phys. A412, 523 (1984)

[6] W. Fetsoher, H.J. Gerber, Adv. Ser. Direct. High Energy Phys. 14, 657 (1995), [,657(1993)]

[7] A.B. Arbuzov, T.V. Kopylova, JHEP 09, 109 (2016), 1605 . 06612

[8] N. Shimizu et al. (Belle), PTEP 2018, 023 C01 (2018), 1709.08833

[9] A. Flores-Tlalpa, G. López Castro, P. Roig, JHEP 04, 185 (2016), 1508 . 01822

[10] J.P. Lees et al. (BaBar), Phys. Rev. D91, 051103 (2015), 1502.01784

[11] T. Kinoshita, J. Math. Phys. 3, 650 (1962)

[12] T.D. Lee, M. Nauenberg, Phys. Rev. 133, B1549 (1964), [,25(1964)]

[13] J. Sasaki (Belle), J. Phys. Conf. Ser. 912, 012002 (2017)

[14] J. Sasaki (Belle), Nucl. Part. Phys. Proc. 287-288, 212 (2017)

[15] Y. Jin, D. Epifanov, H. Aihara, SciPost Phys. Proc. 1, 002 (2019)

[16] A. Blondel et al. (2013), 1301.6113

[17] M. Fael, L. Mercolli, M. Passera, JHEP 07, 153 (2015), 1506.03416

[18] G.M. Pruna, A. Signer, Y. Ulrich, Phys. Lett. B772, 452 (2017), 1705.03782

[19] M. Fael, C. Greub, JHEP 01, 084 (2017), 1611.03726

[20] G.M. Pruna, A. Signer, Y. Ulrich, Phys. Lett. B765, 280 (2017), 1611.03617

[21] J. Kuipers, T. Ueda, J.A.M. Vermaseren, J. Vollinga, Comput. Phys. Commun. 184, 1453 (2013), 1203.6543

[22] R. Mertig, M. Bohm, A. Denner, Comput. Phys. Commun. 64, 345 (1991)

[23] V. Shtabovenko, R. Mertig, F. Orellana, Comput. Phys. Commun. 207, 432 (2016), 1601.01167

[24] T. Hahn, M. Perez-Victoria, Comput. Phys. Commun. 118, 153 (1999), hep-ph/9807565

[25] G.J. van Oldenborgh, J.A.M. Vermaseren, Z. Phys. C46, 425 (1990)

[26] A. Denner, S. Dittmaier, L. Hofer, Comput. Phys. Commun. 212, 220 (2017), 1604.06792

[27] G.P. Lepage, J. Comput. Phys. 27, 192 (1978)

[28] T. Hahn, Comput. Phys. Commun. 168, 78 (2005), hep-ph/0404043

[29] S. Dittmaier, Nucl. Phys. B565, 69 (2000), hep-ph/9904440

[30] T. Bergfeld et al. (CLEO), Phys. Rev. Lett. 84, 830 (2000), hep-ex/9909050

[31] R.R. Crittenden, W.D. Walker, J. Ballam, Phys. Rev. 121, 1823 (1961)

[32] A.M. Baldini et al. (MEG), Eur. Phys. J. C76, 108 (2016), 1312 . 3217

[33] D. Pocanic (PEN) (2015), 1512.09355

[34] M. Tanabashi et al. (Particle Data Group), Phys. Rev. D98, 030001 (2018)

[35] W.H. Bertl et al. (SINDRUM), Nucl. Phys. B260, 1 (1985)

[36] M.S. Alam et al. (CLEO), Phys. Rev. Lett. 76, 2637 (1996) 